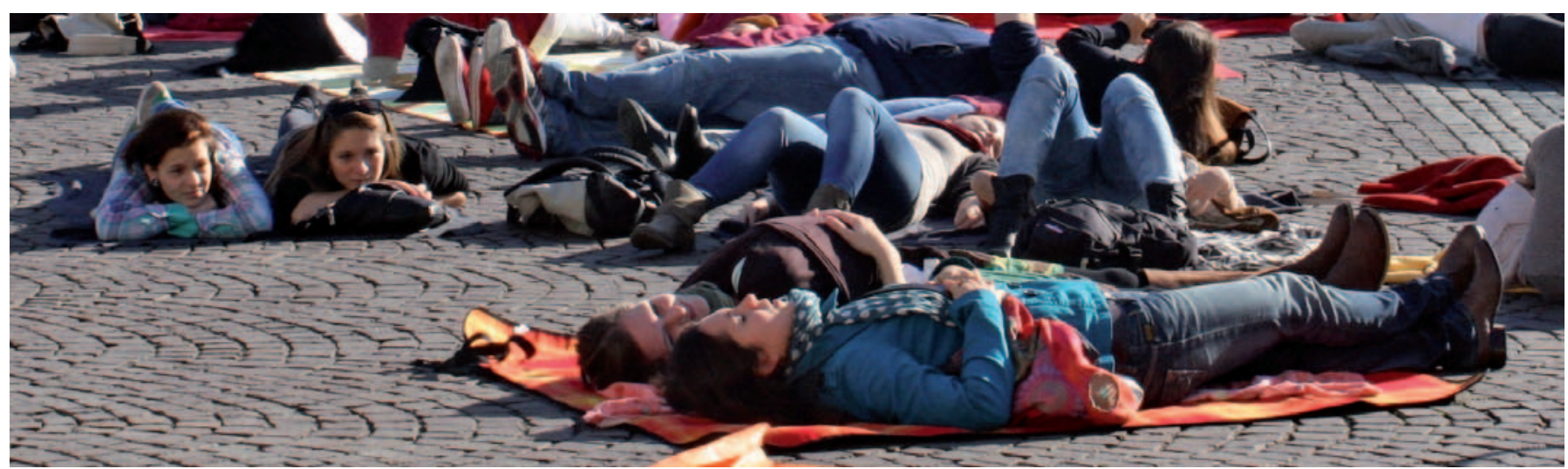

\title{
Die Pflege braucht Pflege
}

„Pflege am Boden“ heißt ein unabhängiger Zusammenschluss von Pflegenden, die sich schon 2013 gemeinsam mit Unterstützern für zehn Minuten lang in vielen Städten Deutschlands symbolisch auf den Boden gelegt haben. Unsere Autorin Franziska Schwalbe fragt sich, wie angesichts der hohen Arbeitsbelastung, der schlechten Arbeitsbedingungen, der geringen Wertschätzung und der fehlenden Stimme in der Politik die Pflege noch zu retten ist.

Franziska Schwalbe

Die Einführung der DRGs (Diagnosis Related Groups) gab den Anstoß für die Entwicklung des Gesundheitswesens zur Gesundheitswirtschaft. Der Patient muss sich lohnen, auch wenn das heißt, immer mehr Patienten in derselben Zeit zu behandeln. Folge dieser Entwicklung ist die Erhöhung der Fallzahlen und die gleichzeitige Reduktion der Kosten, und das oft zulasten des Personals. Doch nicht nur die finanzielle Schieflage vieler Kliniken ist Grund für das Nichtbesetzen notwendiger Planstellen. Unbeliebte Arbeitszeiten, die Unwissenheit über Aufstiegsmöglichkeiten und eine geringe oder falsche Wertschätzung in der Gesellschaft („Ich könnte das nicht“) sind Auslöser für niedrige Ausbildungszahlen.

Welche Möglichkeiten bleiben der Pflege, damit dieses Berufsfeld wieder mehr Wertschätzung in der Gesellschaft erfährt? Welche Rolle spielt die Pflege in der Politik, deren Aufgabe es ist, die Gesundheit der Bevölkerung nachhaltig zu gewährleisten und zu sichern? Und wie kann sich die Pflege auf beruflicher, gesellschaftlicher und politischer Ebene Gehör verschaffen?

\section{Der Weg in eine \\ Gesundheitswirtschaft}

Das Krankenhaus wird als Wirtschaftsunternehmen betrachtet. Viel Kritik geht zulasten des Ökonomisierungsprozesses, der mit der Einführung der Fallpauschalen (DRGs) seinen Anfang nahm. Die Abkehr von den noch bis 2003 bestehenden Tagessätzen - also einer Tagespauschale, die, egal wie lang der Patient im Krankenhaus verblieb, von den Krankenkassen entrichtet wurde - ist Auslöser für das Einführen wirtschaftlicher Instrumente im Gesundheitswesen. Das heißt: Prozesse optimieren, unnötige Tätigkeiten reduzieren, deckungs- und gewinnorientiertes Handeln.

Die Personalkosten machen rund 20\% der Gesamtausgaben eines Krankenhauses aus. ${ }^{1}$ Da liegt die Vermutung nahe, dass im Personalbereich große Möglichkeiten bestehen, um Kosten zu sparen. Hinzu kommen die Reduzierung der Verweildauer, ein schnelleres „Durchschleusen“ der Patienten sowie die Erhöhung der Fallzahl im Krankenhaus, die in den Jahren von 1991 bis 2012 um knapp $28 \%$ gestiegen ist. ${ }^{2}$ In den Ausbau wettbewerblicher Strukturen und Imagekampagnen - vor allem im
Kampf um den „Kunden“ Patient - werden zunehmend höhere Investitionen gesteckt, statt in die Schaffung einer qualitätserhaltenden Personalplanung zu investieren. ${ }^{3}$

\section{Steigende Arbeitsbelastung vs. Fachkräftemangel}

Neben der zunehmenden Arbeitsbelastung aufgrund steigender Fallzahlen führt die Entwicklung demografischer Altersstrukturen $\mathrm{zu}$ einem oft multimorbiden und hochaltrigen Patientengut, das mit immer mehr medizinischer und technischer Ausstattung versorgt werden kann und wird. Doch dieser Altersprozess ist auch im Pflegebereich sichtbar: Im Zeitraum von 2001 bis 2009 ist die Zahl der unter 35-Jährigen von $37 \%$ auf $31 \%$ gesunken. Im Vergleich dazu stieg die Menge der über 50-jährigen Pflegekräfte von fast 17\% auf über $25 \%$ an. ${ }^{4}$ Diese Zahlen decken sich mit den sinkenden Absolventen im Gesundheits- und Krankenpflegebereich: 7.000 Auszubildende fehlten im Jahr 2012 trotz möglicher Ausbildungsstellen. ${ }^{2}$ Ein weiterer Grund für die steigende Arbeitsbelastung sind, v.a. im Intensiv- und Anästhesiebereich, immer größer werdende 
Anforderungen im fachlich-methodischen Tätigkeitsfeld. ${ }^{5}$ „Gute Pflege braucht Zeit“, so die Meinung des derzeitigen Gesundheitsministers Hermann Gröhe, ${ }^{6}$ doch sie braucht auch Qualität, denn Fachkräftemangel bedeutet nicht nur ein Mangel an Pflegepersonal, sondern auch die nicht adäquate Anpassung der Pflegekräfte auf die erhöhten Fallzahlen und an die medizinischen Bedürfnisse und qualitativen Anforderungen des Patienten. ${ }^{7}$

\section{Strukturelle und qualitative Auswirkungen und die Zufriedenheit des Personals}

In einer Studie von 2012 gaben 92\% aller befragten Pflegekräfte an, unter ständigem Zeitdruck zu stehen. ${ }^{8}$ Dies führt, wie in vielen internationalen Studien nachgewiesen, zu einem vermehrten Auftreten medizinischer und hygienischer Mängel, die die Patientensicherheit in einem nicht geringen Maß gefährden. ${ }^{9}$ Eine mangel- bzw. minderqualitative Besetzung im Pflegebereich kann zu Versorgungsmissständen führen und die Zahl der Komplikationen (nosokomiale Infektionen, pulmonale Insuffizienz etc.) und Fehlbehandlungen (z.B. medikamentöse Fehlgaben) während des Krankenhausaufenthalts enorm steigern. ${ }^{10}$

In einer Befragung zur Arbeitsplatzzufriedenheit aus dem Jahr 2011 ist nur etwa die Hälfte aller Pflegekräfte in ihrem Beruf zufrieden und hält die Bedingungen für attraktiv. Neben Faktoren wie Zunahme an Stress, Mangel an Personal und Wechselschichten nennt das befragte Pflegepersonal die geringe Wertschätzung am eigenen Arbeitsplatz als Grund für die Unzufriedenheit. ${ }^{11}$ Hervorzuheben ist hier zum einen die Beziehung zwischen Arzt und Pflegekraft, aber auch die Beziehung der Pflegenden zur zugehörigen Führungsperson, die noch von traditionellen Hierarchiestrukturen gekennzeichnet ist. ${ }^{12}$ Wertschätzung in Form von Dankbarkeit und Lob erfahren Pflegende meist nur vonseiten der Patienten, wenn diese sich mitfühlend gepflegt und gut versorgt sehen.

\section{Die Wertschätzung der Pflege}

Die Steigerung der Anerkennung des Pflegeberufs in der Öffentlichkeit steht dem mangelnden Interesse der Gesellschaft im Weg. Wer möchte sich schon gern über

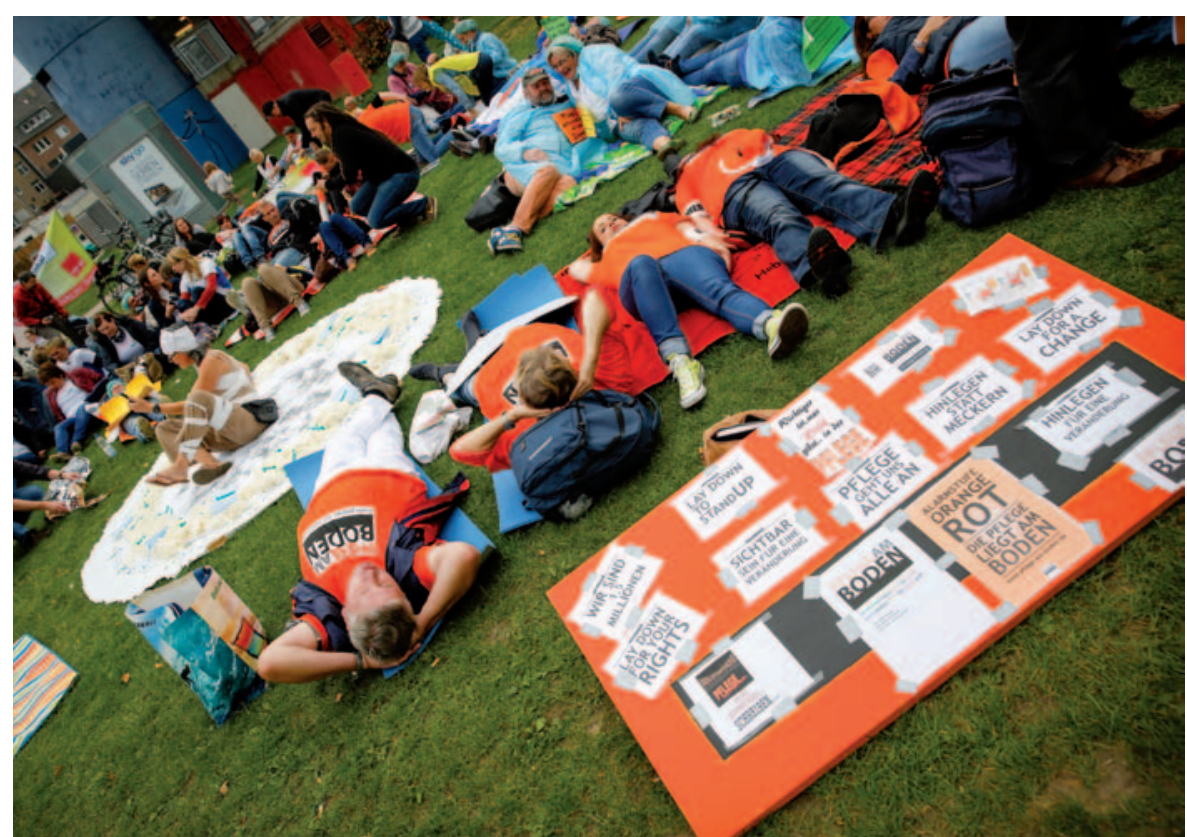

Seit dem ersten Smartmob „Pflege am Boden“ im Oktober 2013 haben sich in über 150 Städten mehr als 120.000 Menschen auf die Straße gelegt und auf die Missstände in der Pflege aufmerksam gemacht. Auch 2015 wird die Aktion fortgesetzt.

das Thema Krankheit Gedanken machen und sich mit den Missständen in der Klinik auseinandersetzen, wenn man nicht mit deren Inanspruchnahme in Berührung kommt? Das mangelnde Wissen über die Notwendigkeit genügender Pflegekräfte, deren Tätigkeitsbeschreibungen und fehlende Kenntnisse über Spezialisierungen im Pflegebereich sind Gründe dafür, warum die Pflege im Allgemeinen wenig Wertschätzung in der Gesellschaft erfährt. ${ }^{13}$ Das erhöht die Frustration des Pflegepersonals, denn wie kann die eigene Tätigkeit in vollem Maße wertgeschätzt werden, wenn dies nicht auch in ausreichendem Maße im externen Umfeld stattfindet? ${ }^{14,15}$

\section{Die Pflege im Gespräch - Lösungswege von der Politik}

Die Maßnahmen zur Verbesserung der Pflege beschränkten sich in den letzten Jahren im Wesentlichen auf die Sicherstellung der pflegerischen Versorgung, die Verbesserung der Arbeitsbedingungen und die Steigerung der Attraktivität des Pflegeberufs. ${ }^{16}$ Doch die konträren Meinungen der Parteien verhinderten eine Ausgestaltung der schon damals zur Sprache gebrachten Pflegereform. ${ }^{17}$ Im derzeitigen
Koalitionsvertrag nimmt die Bedeutung der Pflege wieder eine wichtige Rolle ein, jedoch in erster Linie zur Verbesserung der Situation im Bereich der Altenpflege. ${ }^{18}$ Die Ursachen, die zum Fachkräftemangel beitragen, treten trotz Beachtung der Pflege in der 18. Legislaturperiode in den Hintergrund. Die geringe Wertschätzung und die organisatorischen Defizite in der Tätigkeitsbeschreibung und Personalbemessung bleiben weiter unbeachtet. Infolgedessen erläuterte Rudolf Henke (MdB, CDU/CSU) in einem Gespräch in Berlin, dass bei Einführung der DRGs im Jahr 2003 das Abbilden des Pflegeaufwands und der -qualität in deutschen Kliniken zwar Thema war, dass dem jedoch nicht viel Beachtung geschenkt wurde. ${ }^{19}$ Es haben sich nach über zehn Jahren lediglich Standardisierungen im pflegerischen Prozess sowie die dokumentarischen Aufgaben erhöht. Die Etablierung eines neuen Qualitätsinstituts ist ebenfalls Teil des aktuellen Koalitionsvertrags und soll mehr Transparenz auch im Pflegebereich schaffen. ${ }^{20}$ Elisabeth Scharfenberg (MdB, Bündnis 90/Die Grünen) geht in diesem Zusammenhang auf die Problematik der mangelnden Qualitätsmessung im Pflegebereich ein. Weiterhin sagt sie: „Vieles liegt in der Hand 


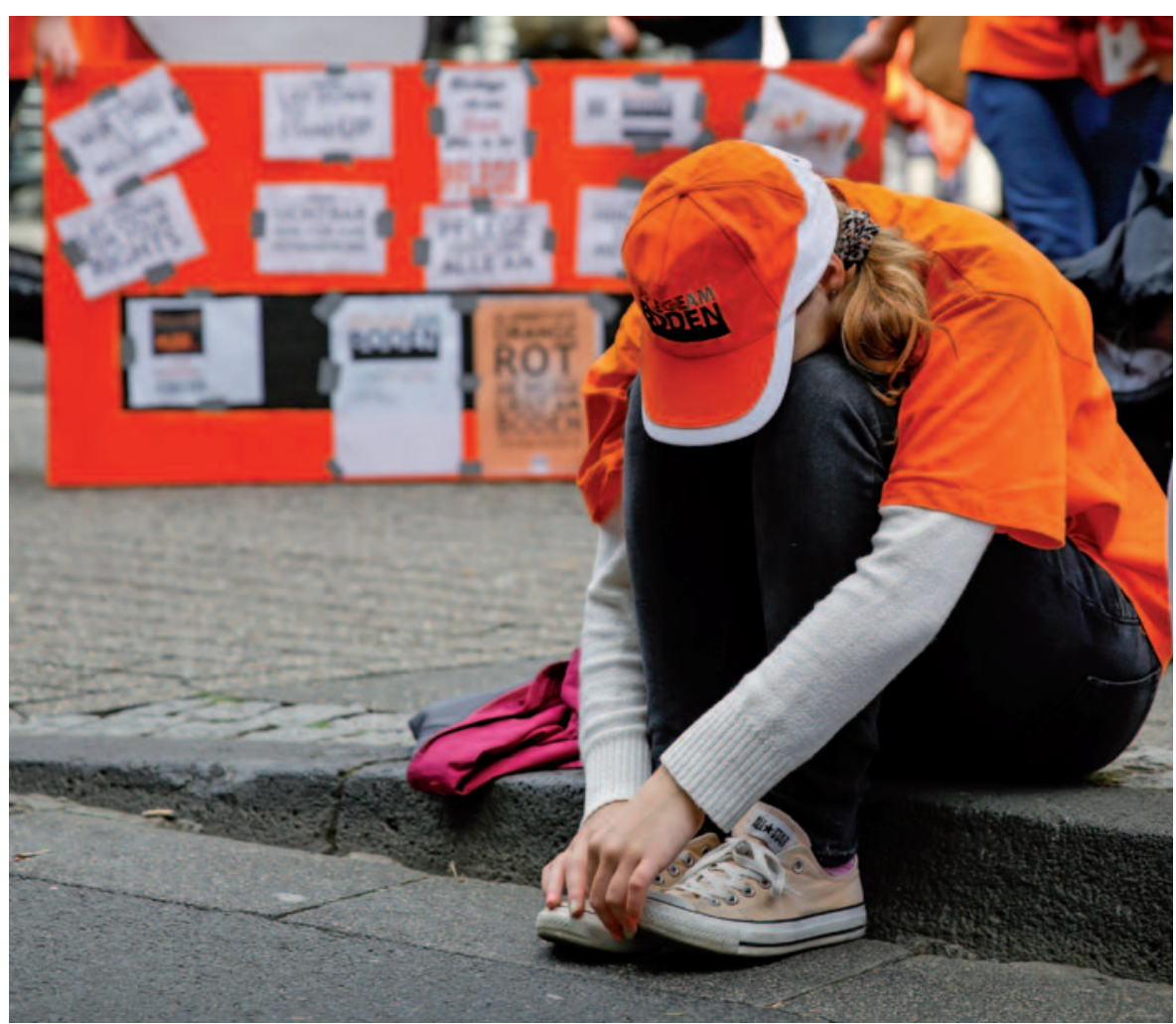

Zu niedrige Bezahlung, geringe Wertschätzung der Tätigkeit, erhöhter Dokumentationsaufwand, immer weniger Zeit für Fürsorge und Betreuung - die Situation der Pflegenden verschlechtert sich stetig.

der (...) Krankenhäuser. Deshalb sind hier auch die Arbeitgeber gefragt, denn sie können vieles verändern. (...) Auch die Arbeitnehmer selbst sind gefragt. (...) Ein hohes Maß an Loyalität, Einsatzbereitschaft und Verantwortungsbewusstsein (...) ist aber durchaus auch ein Teil des Problems."21 Damit spricht sie die mangelnde Selbstorganisation der Pflege an. Groß im Gespräch ist auch die Vereinheitlichung der Pflegeausbildung. So ist eine einheitliche Ausbildung für Gesundheits- und (Kinder-)Krankenpflege sowie Altenpflege mit nachfolgender Spezialisierung geplant. Andreas Westerfellhaus (Präsident des Deutschen Pflegerats) hält dies für eine Möglichkeit, mehr Auszubildende für den Pflegeberuf zu begeistern. Dies wird aber dem Personalmangel nicht ausreichend entgegenwirken können. ${ }^{22}$ Damit wird ein weiteres Problem angesprochen: die Diskrepanz zwischen einer Akademisierung der Pflege und dem schnellen Rekrutieren von neuem, meist nicht ausreichend qualifizierten Personal.

\section{Der Ausweg - eine wertgeschätzte Pflege}

Bei den Auswirkungen der ökonomischen Einflüsse auf das Krankenhauswesen handelt es sich hauptsächlich um die Arbeitsverdichtung und die gleichzeitige nicht an die Umstände angepasste Personalstruktur. Dies führt dazu, dass Pflegekräfte in vielen Fällen keine Zeit mehr haben, ihre Arbeit in einer idealistischen Vorstellung durchzuführen, geschweige denn nach hygienischen Richtlinien. Es entsteht eine Art Zielkonflikt: Die notwendigen Leistungen können in der vorgegebenen Zeit nicht erfüllt werden. ${ }^{23}$ Denn Pflege heißt nicht ausschließlich eine der medizinischen Situation des Patienten angepasste Versorgung durchzuführen. Professionell pflegen bedeutet umfassend zu versorgen und in einem gewissen Maße die Bedürfnisbefriedigungen auf emotionaler und psychischer Ebene seitens des Patienten miteinzubeziehen. Doch auch viele Pflegende sind sich dieser Tatsache nicht in dem Maße bewusst, als dass sie dies auch in die Öffent- lichkeit hinaustragen, und verstärken somit nur die unzureichende Wertschätzung in der Gesellschaft. Denn nur ein geringer Anteil der Pflegekräfte würde ihren Beruf weiterempfehlen - das zeigen Ergebnisse einer Befragung von mehr als 3.000 Pflegekräften der Fachhochschule Münster. ${ }^{24}$ Die prekäre Situation führt zum sinkenden Bewusstsein über die Wichtigkeit, die Vielfältigkeit und den wertschätzenden Inhalt der Tätigkeit. ${ }^{14,15}$ Aber wie soll die Pflege Anerkennung auf beruflicher, gesellschaftlicher und politischer Ebene erfahren, wenn sie selbst nicht von der Wichtigkeit ihrer Tätigkeit in vollem Maße überzeugt ist?

\section{Die Pflegekammer \\ als Lösung des Problems?}

Die Regierung auf Regional-, Länder- und Bundesebene ist Rahmengeber für arbeitsrechtliche Bedingungen und Strukturen. Um Veränderungen in diesem Bereich zugunsten der Pflege herbeizuführen bedarf es einer Stimme im politischen Meinungsprozess. Etliche Berufsverbände verfolgen bereits diesen Weg, um die Interessen der Pflegenden auf allen Ebenen zu vertreten. Um politischen Einfluss nehmen zu können, bedarf es einer großen Mitgliederzahl in den Verbänden, doch ist nur ein Teil der Pflegekräfte gewillt, Beiträge dafür zu entrichten, dass ihre Interessen vertreten werden. Die Einführung einer Pflegekammer steht in vielen Bundesländern zur Debatte. Lediglich Schleswig-Holstein ist Vorreiter in der Einführung einer Pflegekammer.

Als Vorteile der Einführung einer Pflegekammer werden genannt:26

- die Erhöhung der Versorgungsqualität, ${ }^{25}$

- die Förderung der Arbeitsbedingungen,

- Definition der Inhalte und des Tätigkeitsspektrums der Pflege,

- das Erarbeiten einer einheitlichen Ausbildungs- und Prüfungsordnung sowie der Fachweiterbildungen,

- mehr Autonomie des Pflegeberufs, Beitrag zur Professionalisierung,

- Öffentlichkeitsarbeit und Vertretung im politischen Meinungsbildungsprozess.

Im Gegenzug dazu ist den Pflegenden laut Elisabeth Scharfenberg oft nicht klar, „(...) 
welche Ziele, Aufgaben und auch Erwartungen eine Kammer erfüllen kann und welche nicht. So kann die Einrichtung einer Kammer eventuell ein Schritt zur Statusangleichung an andere Berufsgruppen sein. (...) Es ist auch zu überlegen, ob eine Gesundheitskammer für alle nichtärztlichen Gesundheitsberufe möglicherweise zielführender und schlagkräftiger wäre als eine reine Pflegekammer.“27 Neben der Verbesserung des Einflusses der Pflege auf den politischen Meinungsprozess sind Punkte wie die Vereinheitlichung von inhaltlichen Aus- und Weiterbildungsstrukturen und somit vielleicht eine Anpassung des Entgeltsystems an höher qualifizierte Pflegekräfte denkbare Vorteile. Die Pflichtmitgliedschaft und die daraus entstehenden Beiträge stellen für viele Pflegekräfte aber einen Kritikpunkt dar.

Jedoch muss angemerkt werden, dass eine Selbstorganisation der Pflege, egal in welcher Form, Raum gibt, um aussagekräftige Daten über Qualitätszustände, Personalbemessungen und Personalbedarf zu sammeln und auszuwerten. ${ }^{28}$ Die Pflege muss lernen, für sich und ihre Interessen einzutreten, denn nur so kann eine Aufwertung des Berufsbilds erfolgen und ein hochrangiger Wert im Berufsumfeld, in der Gesellschaft und Politik erreicht werden. Erst wenn die Wichtigkeit des Berufszweigs erkannt wird, verbessern sich Arbeitsbedingungen und die Entgeltsituation. Die Pflege muss Pflege für sich selbst betreiben - denn wenn sich die Pflege nicht für sich interessiert, wer interessiert sich dann dafür?

\section{Literatur}

1 Statistisches Bundesamt: Gesundheit. Kostennachweis der Krankenhäuser. Fachserie 12 Reihe 6.3., 2013

2 Statistisches Bundesamt: Gesundheit. Grunddaten der Krankenhäuser. Fachserie 12 Reihe 6.1.1., 2013

3 Bruckenberger E, Klaue S, Schwintowski HP. Krankenhausmärkte zwischen Regulierung und Wettbewerb. Berlin, Heidelberg, New York: Springer; 2006: 9

4 Simon M. Beschäftigte und Beschäftigungsstrukturen in Pflegeberufen. Eine Analyse der Jahre 1999 bis 2009. Studie für den Deutschen Pflegerat. Fakultät Diakonie Gesundheit und Soziales; 2012: 50

5 Nock L, Hielscher V, Kirchen-Peters S. Dienstleistungsarbeit unter Druck: Der Fall Krankenhauspflege. Hans-Böckler Stiftung; 2013: 27
6 Bundesministerium für Gesundheit: Pressemitteilung Herman Gröhe: „Gute Pflege braucht Zeit“. Abschlussbericht zur Vereinfachung der Pflegedokumentation, veröffentlicht. Online unter www.bmg.bund.de/ministerium/presse/ pressemitteilungen/2014-02/vereinfachungder-pflegedokumentation, letzter Zugriff 21.06.2014

7 Nock L, Hielscher V, Kirchen-Peters S. Dienstleistungsarbeit unter Druck: Der Fall Krankenhauspflege. Hans-Böckler Stiftung; 2013: 26

8 Nock L, Hielscher V, Kirchen-Peters S. Dienstleistungsarbeit unter Druck: Der Fall Krankenhauspflege. Hans-Böckler Stiftung; 2013: 24ff

9 Lindenthal M, Hölscher UM. Patientensicherheit und widersprüchliche Arbeitsanforderungen im Krankenhaus. Zentrum für Medizintechnik und Ergonomie; 2014: 1

10 Simon M. Stellenabbau im Pflegedienst der Krankenhäuser: Mindestanforderungen als Ansatz zur nachhaltigen Sicherung einer ausreichenden Personalbesetzung. Hans-Böckler-Stiftung; 2008: $38 \mathrm{f}$

11 Buxel H. Jobwahlverhalten, Motivation und Arbeitsplatzzufriedenheit von Pflegepersonal und Auszubildenden in Pflegeberufen. Ergebnisse dreier empirischer Untersuchungen und Implikationen für das Personalmanagement und -marketing von Krankenhäusern und Altenpflegeeinrichtungen. Fachhochschule Münster; 2011: 5

12 Glaser J. Arbeitsorganisation im Krankenhaus. S. 32. In: Herbig B, Büssing A. Informations- und Kommunikationstechnologien im Krankenhaus. Grundlagen, Umsetzung, Chancen und Risiken. Stuttgart: Schattauer; 2006: 29-42

13 Kumbruck C, Rumpf M, Senghaas-Knobloch E. Zukünfte des Ethos fürsorgliche (Pflege-)Praxis. Ein Resümee. S. 330. In: Krumbruck C, Rumpf M, Senghass-Knobloch E. Unsichtbare Pflegearbeit - Fürsorgliche Praxis auf der Suche nach Anerkennung. Münster: LIT Verlag; 2010: 323-352

14 Weber H. Die Ambivalenz in der Pflege auf dem Professionalisierungsweg - Eine empirische Untersuchung bei der täglichen Visite. Veröffentlichte Dissertation; 2011: 107

15 Hofmann I. Die Rolle der Pflege im Gesundheitswesen. Historische Hintergründe und heutige Konfliktkonstellationen. Bundesgesundheitsbl 2012, 55: 1161-1167

16 Bundesministerium für Gesundheit: Wir kümmern uns um die Menschen 2011 ist das Jahr der Pflege. Online unter: www.bmg.bund.de/ministerium/presse/ pressemitteilungen/2011-02/2011-jahr-derpflege.html, letzter Zugriff 28.05.2014

17 Rudzio K. 2011 - das Jahr des Stillstands bei der Pflege? Zeit Online Wirtschaft. Online unter: www.zeit.de/2011/39/Pflege-Politik, letzter Zugriff 05.06.2014

18 Bundesministerium für Gesundheit: Richtlinien für die Gesundheitspolitik. Online unter: www.bmg.bund.de/ministerium/presse/reden/ richtlinien-fuer-die-gesundheitspolitik.html, letzter Zugriff 05.06.2014
19 Eigene Befragung des MdB Rudolf Henke (CDU/ CSU) am 21. Mai 2014 zum Thema Abbilden der Pflegequalität im DRG-Fallpauschalen-System

20 Bundesministerium für Gesundheit: Qualitätsinstitut für ambulante und stationäre Behandlung. Online unter: www.bmg.bund.de/ krankenversicherung/finanzierungs-undqualitaetsgesetz/qualitaetsinstitut.html, letzter Zugriff 05.06.2014

21 Eigene Befragung der MdB Elisabeth Scharfenberg (Bündnis 90/Die Grünen) am 21. Mai 2014 zum Thema Selbstorganisation der Pflege

22 Eigene Befragung des Präsidenten des DPR Andreas Westerfellhaus am 27. Mai 2014 zum Thema der Vereinheitlichung der Pflegeausbildung und der Personalsituation in deutschen Krankenhäusern

23 Lindenthal M, Hölscher UM. Patientensicherheit und widersprüchliche Arbeitsanforderungen im Krankenhaus. Zentrum für Medizintechnik und Ergonomie; 2014

24 Buxel H. Jobwahlverhalten, Motivation und Arbeitsplatzzufriedenheit von Pflegepersonal und Auszubildenden in Pflegeberufen. Ergebnisse dreier empirischer Untersuchungen und Implikationen für das Personalmanagement und -marketing von Krankenhäusern und Altenpflegeeinrichtungen. Fachhochschule Münster; 2011: 427

25 Eigene Befragung der MdB Elisabeth Scharfenberg (Bündnis 90/Die Grünen) am 06. Juni zum Thema Einführung einer Pflegekammer

26 Nationale Konferenz zur Errichtung von Pflegekammern in Deutschland: Nutzen einer Kammer für den Berufsstand Pflege. Online unter: www.pflegekammer.de/nutzen.htm, letzter Zugriff 20.06.2014

27 Eigene Befragung der MdB Elisabeth Scharfenberg (Bündnis 90/Die Grünen) am 06. Juni 2014 zum Thema Einführung einer Kammer für Gesundheitsberufe

28 Deutscher Berufsverband für Pflegeberufe (DBfK): Informationen zur Pflegekammer. Online unter: www.pflegekammer-jetzt.de/index. php?option $=$ com_contentEview $=$ articleEid $=10$ EItemid=9, letzter Zugriff 07.06.2014

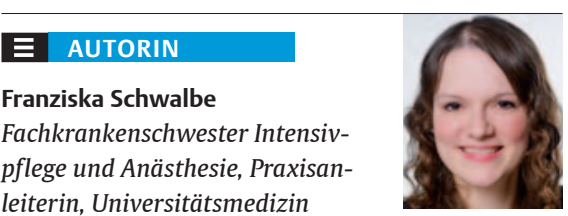
leiterin, Universitätsmedizin Göttingen (UMG). Studentin BA Gesundheitsmanagement an der SRH Riedlingen.

BIBLIOGRAFIE

DOI 10.1055/s-0035-1547198

Intensiv 2015; 23 (2): 64-67

(c) Georg Thieme Verlag KG

Stuttgart · New York · ISSN 0942-6035 